\title{
BASES PARA EL DESARROLLO DE UN PROYECTO FORESTAL CON PLANTACIONES DE ÁLAMOS EN EL VALLE IRRIGADO DE GOBERNADOR GREGORES, ARGENTINA.
}

Davel, M.M. ${ }^{1(4)}$; Fernández, M.V. ${ }^{1(4)}$; Gabriel, J.C.2; Núñez, H.R. ${ }^{(2)}$; Peri, P. ${ }^{(3)}$, Alonso, M.V. ${ }^{1(4)}$

RESUMEN

En la región Patagónica, la ganadería es la principal actividad económica desde hace más de un siglo. Hoy existen, en el área de estudio, alrededor de 1.000 ha sistematizadas para riego con potencial para el cultivo de frutas finas, hortalizas y pasturas. La zona presenta fuertes vientos que tienen efectos nocivos sobre los cultivos, el ganado y el suelo, disminuyendo el nivel de productividad.

La instalación de cortinas protectoras permitiría mejorar la producción agropecuaria en cantidad y calidad. En este contexto se desarrolló el presente proyecto, que tuvo como objetivo general realizar un plan de desarrollo forestal en Gobernador Gregores (zona centro de la provincia de Santa Cruz), a partir de la instalación de cortinas de salicáceas asociadas a las actuales actividades productivas.

El proyecto se dividió en seis etapas que consistieron en la realización de un diagnóstico socio-productivo, la zonificación del área potencial en clases de aptitud para forestar, el análisis del crecimiento de las cortinas en cada una de las clases, la instalación de plantaciones demostrativas, la estimación del incremento de la rentabilidad predial debido a la incorporación de cortinas y una propuesta de desarrollo forestal para el área.

El diagnóstico socio - productivo determinó que las actividades agrícolas y ganaderas se desarrollan en forma complementaria a las urbanas y extractivas. El tamaño promedio de las chacras es de 60,4 ha y las principales actividades productivas son la cría extensiva de ganado ovino, la producción de pasturas y, en menor medida, de fruta fina y hortalizas. Estos productos se comercializan en la zona, con excepción de algunas manufacturas de tipo agropecuario, como por ejemplo la producción de ajo y lana, que trascienden la provincia. La actividad forestal es reducida y se limita a la producción de plantas que se comercializan principalmente en otras zonas de la provincia. Además se determinaron los factores que favorecen y obstaculizan el desarrollo de la zona.

En el área de estudio de 4.822 ha, se determinó una superficie forestal potencial de 2.387,4 ha, que se clasificó en dos clases de aptitud para el cultivo de salicáceas; apta (36\%) y

\footnotetext{
1 Centro de Investigación y Extensión Forestal Andino Patagónico (CIEFAP). Ruta 259 km 4 CC14. Esquel, Chubut. Investigadores.mdavel@ciefap.org.ar ; mvfernandez@ciefap.org.ar

2 Consejo Agrario Provincial. Av. Roca 976, Río Gallegos, Santa Cruz. juancarlosgabriel@hotmail.com.ar; desarrollolocal@argentina.com

3 INTA Santa Cruz. pperi@correo.inta.gov.ar

4 Universidad Nacional de la Patagonia San Juan Bosco - Ruta 259 km 4. Esquel, Chubut. Docentes
}

Volumen 16 № 3, Diciembre 2010 / 331 
medianamente apta (64\%). Los valores promedio de crecimiento en altura fluctúan en torno a $45 \mathrm{~cm} /$ año y en volumen $3 \mathrm{~m}^{3} / \mathrm{año} \mathrm{cada} 100 \mathrm{~m}$ de cortina.

Los cultivares que presentaron mejores comportamientos son: Populus nigra 'Italica'; Populus nigra 'Moissac' y Salix matsudana $x$ alba RA 524-43. Se definió el diseño de cortinas para cada tipo de cultivo y en base a ello se realizaron plantaciones demostrativas y se analizaron indicadores de rentabilidad y de flujo de caja incremental, por la incorporación de cortinas asociadas a la producción de pasturas. EI VAN incremental resultó positivo en todos los casos.

Con toda la información generada se diseñó una propuesta de desarrollo forestal, factible desde el punto de vista socio-económico, que implica la forestación de 100 ha/año con cortinas de salicáceas con fines de protección, durante los primeros 17 años. Esto implica instalar 11.000 metros de cortinas por año.

A partir de los 39 años, se estima que estas cortinas aportarán como producción secundaria, unos $6.500 \mathrm{~m}^{3} /$ año de madera rolliza y unos $2.500 \mathrm{~m}^{3} /$ año de leña y permitirán la expansión de la actividad agrícola en forma sustentable.

Palabras clave: Populus nigra Italica; Populus nigra Moissac y Salix matsudana x alba RA 524-43, Patagonia. 


\section{BASIS FOR THE DEVELOPMENT OF A FOREST PROJECT WITH POPLAR PLANTATIONS IN THE GOBERNADOR GREGORES IRRIGATED VALLEY, ARGENTINA.}

\section{SUMMARY}

In Argentine Patagonia, livestock grazing has been the main economic activity during the last century. Today, there are about one thousand hectares systematized for irrigation activities with potential cultivation of berries, vegetables and pastures. The whole region presents strong winds which have damaging effects upon crops, animals and soils, by diminishing their productivity.

The establishment of wind breaking barriers could be a mean to improve crops and livestock production either in quantity and quality. Within this context, this project was developed with the general objective of carry out an afforestation development plan in Gobernador Gregores (center of Santa Cruz Province), based on the establishment of wind breaking barriers (Populus and Salix spp.) associated to areas with existing productive activities.

The project was divided into six phases, which consisted in making a diagnosis about the socio-productive activities, subdivision of areas in different afforestation classes, analyze the growth capabilities of wind braking barriers within each defined class, installation of demonstrative plantations, economic analysis of rentability of each farm due to the incorporation of wind breaking barriers, and to make a forest development proposal for the area.

The socio-productive diagnosis determined that crop and livestock activities are developed complementary to urban and extractive economic activities. The mean size of the farms is $60.4 \mathrm{ha}$, and their main activities are extensive sheep and pasture production, and to a lesser degree fruit and vegetables production. These products are marketed into the surrounding zone with the exception of wool and garlic that are marketed beyond the provincial limits. Forest activity is limited and reduced to tree seedling production that generally is marketed into other areas of the same province. In this diagnosis are also determined the factors that favor or hinder the economic development of the area.

Within the study area, which comprised 4,822 ha, was determined that 2,387.4 ha have an afforestation potential, of which $36 \%$ are very suitable for the cultivation of Populus and Salix spp., while the rest (64\%) is relatively suitable for Populus and Salix spp. cultivation. The mean growth values in height are around $45 \mathrm{~cm} /$ year, and in volume close to $3 \mathrm{~m}^{3} / y e a r$, for every $100 \mathrm{~m}$ of wind break barrier.

The best growth behavior was obtained with Populus nigra 'Italica'; Populus nigra 'Moissac' and Salix matsudanax alba RA 524-43. The design of the wind breaking barriers was defined for each kind of plantation and based on that demonstrative trials were established and the economic analyses of rentability due to the incorporation of these barriers was done. The VAN was positive in all cases. 
With the generated information an economically feasible forest plan that implies the afforestation of 100 ha/year for the next 17 years was determined. These numbers mean to plant $11,000 \mathrm{~m}$ of wind breaking barriers per year.

It is estimated that from the year 39 after plantation, these wind breaking barriers would produce $6,500 \mathrm{~m} 3$ of round wood and about 2,500 m3 of firewood per year, and would allow to expand agricultural activities in a sustainable way.

Key Words: Populus nigra Italica; Populus nigra Moissac and Salix matsudana $x$ alba RA 52443. Patagonia

\section{INTRODUCCIÓN}

La zona de estudio se caracteriza por un clima semiárido con fuertes vientos, donde el déficit hídrico puede ser compensado en parte mediante el riego. Sin embargo esta práctica, para que sea eficiente, debe ser acompañada con una adecuada protección mediante la instalación de cortinas cortaviento.

En el valle existe una superficie cercana a las 1.000 ha que está sistematizada para riego. También existen algunos pocos ejemplos, pero muy demostrativos, de predios con riego, cortinas y producción de pasturas, hortalizas y algunos frutales que contrastan fuertemente con la gran mayoría de los predios no forestados.

En este marco, la incorporación de la actividad forestal se presenta como una alternativa para revertir los procesos de desertificación, aumentar y diversificar la producción, enriquecer el paisaje y al mismo tiempo incrementar las fuentes de empleo. Además, si estas cortinas son adecuadamente manejadas brindarán, como beneficio adicional, la posibilidad de extraer madera y leña.

En los últimos años la población de Gobernador Gregores se ha incrementado en forma importante por la actividad minera, basada en un recurso que no es renovable y, por lo tanto, es limitado en el tiempo. Esto lleva a que se deban buscar alternativas que cubran, aunque sea en parte, los efectos de la culminación de la minería.

En el contexto indicado, la actividad agroforestal se presenta como una alternativa válida y sustentable. La instalación de cortinas forestales se ve favorecida además por la existencia de subsidios nacionales que se entregan a los productores que forestan y a la existencia de conocimientos científicos y técnico para la producción del material de plantación, el establecimiento de las plantaciones y su manejo posterior.

En base a esta situación se desarrolló este proyecto entre el CIEFAP y el Consejo Agrario Provincial de Santa Cruz (CAP), que tuvo como objetivo general realizar un plan de desarrollo forestal del área a partir de la instalación de cortinas de salicáceas asociadas a las actuales actividades productivas. El mismo cuenta con la colaboración de personal de la Fundación Bosques de la Patagonia, la Universidad Nacional de la Patagonia Austral (UNPA), la Universidad Nacional de la Patagonia San Juan Bosco (UNPSJB), el INTA, la Escuela 
Agrotécnica y productores de Gobernador Gregores, y es financiado por SECTyP. El proyecto constó de seis etapas:

- $\quad$ Diagnóstico de la situación socio-productiva de propietarios o grupos de propietarios con disponibilidad de tierras con potencial para el establecimiento de plantaciones bajo riego.

- $\quad$ Determinación del área potencial para forestar con salicáceas y zonificación de la misma por clases de aptitud.

- $\quad$ Evaluación de los crecimientos de las plantaciones existentes en el área.

- Instalación de plantaciones demostrativas.

- $\quad$ Estudio de viabilidad económica para el establecimiento de plantaciones bajo riego.

- $\quad$ Propuesta de desarrollo forestal para el área y acciones de transferencia.

\section{MATERIAL Y MÉTODO}

\section{Primera Etapa. Diagnóstico de la situación socio-productiva de productores con disponibilidad de tierras para el establecimiento de plantaciones bajo riego}

Para la realización del diagnóstico se hizo un relevamiento de datos primarios a través de una encuesta semi-estructurada realizada a los productores. Esta información se complementó con datos secundarios obtenidos de material bibliográfico y estadístico relacionado y trabajos sobre el tema realizados con anterioridad.

Para el trabajo de campo se realizó un muestreo de tipo no probabilístico. La unidad de análisis la constituyeron aquellos productores con disponibilidad de suelos aptos para la forestación con salicáceas. La muestra estuvo constituida por 32 propietarios, lo que representa alrededor del $60 \%$ de los productores. Respecto del área de cobertura, la muestra representó el $40 \%$ del total del área de estudio y aproximadamente el $81 \%$ de la que comprende la zona apta para plantar álamos.

\section{Segunda Etapa. Determinación del área potencial para forestar con salicáceas y zonificación por clases de aptitud}

En un estudio previo, a partir de imágenes satelitales, se determinó la superficie del área de estudio de 4.348 ha y, dentro de la misma, la superficie potencial para forestar con salicácea de 2.583,6 ha (Davel et al., 2007). Sobre esta última superficie se realizó un chequeo a campo y se tomaron datos para la zonificación por clases de aptitud. Para ello se recorrió el área tratando de cubrir las distintas situaciones que se presentaban, teniendo en cuenta principalmente el tipo de vegetación y las especies presentes.

En cada punto de muestreo se obtuvo una muestra de suelo, entre los 30 y $60 \mathrm{~cm}$ de profundidad, para realizar los análisis en laboratorio $(\mathrm{pH}$, salinidad, contenido de materia orgánica, nitrógeno total, fósforo disponible y potasio asimilable). Además, se determinó la 
profundidad efectiva con un barreno de suelos y la textura al tacto y se tomó información de altitud, latitud y longitud con GPS. En total se establecieron 82 puntos de muestreo distribuidos en los predios de 45 productores. Esta información fue cargada en un sistema de información geográfica. En el Cuadro $N^{\circ} 1$ se presenta un resumen de la información obtenida en todos los puntos de muestreo.

\section{Cuadro $\mathrm{N}^{\circ} 1$}

\section{ESTADÍSTICOS DESCRIPTIVOS DE LAS VARIABLES DE SITIO MEDIDAS EN EL TRABAJO DE CAMPO}

\begin{tabular}{|c|c|c|c|c|}
\hline Variable & Promedio & Máximo & Mínimo & $\begin{array}{l}\text { Desviación } \\
\text { Estándar }\end{array}$ \\
\hline Profundidad efectiva del suelo (m) & 0,7 & 1 & 0,2 & 0,2 \\
\hline Pedregosidad (\%) & 1,8 & 33 & 0 & 5,3 \\
\hline Altitud (msnm) & 283 & 305 & 265 & 9,4 \\
\hline Latitud & & 48ㄴㅇ․ㄴ" & $48^{\circ} 44^{\prime} 15,6^{\prime \prime}$ & \\
\hline Longitud & & $70^{\circ} 29^{\prime} 15,8^{\prime \prime}$ & 7010’33”' & \\
\hline Pendiente (grados) & 0,1 & 2 & 0 & 0,3 \\
\hline $\mathrm{pH}$ & 7,8 & 8,7 & 6,0 & 0,6 \\
\hline Salinidad (conductividad dS/m) & 0,6 & 2,7 & 0,1 & 0,5 \\
\hline Materia Orgánica (\%) & 1,7 & 2,9 & 0,7 & 0,5 \\
\hline N (\% N Total) & 0,09 & 0,15 & 0,05 & 0,04 \\
\hline P disponible (mg/kg) & 23,18 & 66 & 3 & 16,24 \\
\hline $\mathrm{K}$ asimilable $(\mathrm{mg} / \mathrm{kg})$ & 92,22 & 283 & 35 & 54,33 \\
\hline
\end{tabular}

En cuanto a la textura, el $33 \%$ de los sitios presentaban suelos franco arcillosos a arcillosos, el 39\% textura franca a franca arenosa y el resto corresponde a suelos donde predominan arena y arcilla mezcladas (arcillo arenosos a franco arcillo arenosos).

Posteriormente y en base a información bibliográfica (FAO, 1980; Riu et al., 1988; Suarez, 1993; Menoyo et al., 1994; Deschamps y Wright, 1997; Amico, 2001; García, 2002), cada punto fue clasificado según su aptitud en:

- Sitios no aptos: Se consideraron así aquellos que presentan problemas de salinidad (conductividad eléctrica mayor a 0,9 dS/m), suelos sódicos (PSI igual o mayor a 15\%), suelos muy pesados (arcillosos) y poco profundos (menos de 0,50 m). En estas condiciones estas especies no pueden desarrollarse.

- Sitios medianamente aptos: Se clasificaron en esta categoría aquellos que no tienen problemas de salinidad ni sodicidad, pero que tienen alguna otra limitante como alto 
porcentaje de arcilla, pH elevado, pedregosidad, otras. En ellos los álamos desarrollan pero sus crecimientos son muy lentos.

- Sitios aptos: Son los que presentan suelos profundos (>0,80 m), textura franca y sin ninguna de las limitantes mencionadas anteriormente. En ellos los álamos alcanzan los mejores crecimientos en la zona.

\section{Tercera Etapa. Crecimiento de las plantaciones existentes dentro del área potencial y para cada clase de aptitud de sitio}

Para estudiar el crecimiento se tomó información en el terreno midiendo plantaciones presentes en las distintas clases de aptitud y se recopilaron trabajos existentes en la zona sobre este tema (Peri et al., 1997; Peri y Monelos, 1998; Peri y Martínez Pastur, 1998). En total se tomó información de 39 cortinas, de las cuales 21 se ubicaron en sitios medianamente aptos, 17 en sitios aptos y una sola cortina en un sitio no apto.

Para este análisis, si bien se midieron cortinas de diferentes especies, solo se consideraron las cortinas de álamo criollo por ser la especie presente mayoritariamente en el área $(80 \%$ de las cortinas). En cada una de las cortinas se instaló una parcela lineal que incluyera 20 árboles y, en cada uno de ellos, se midió el DAP (diámetro a la altura del pecho) con cinta diamétrica y la altura total con clinómetro. La edad se determinó sobre tarugos extraídos con un barreno de Pressler. El volumen individual se estimó con la ecuación regional ajustada por Peri y Martínez Pastur (1998) para álamo criollo.

Con esta información se determinaron los crecimientos medios en diámetro, altura y volumen. Por último, de cada cortina, a partir de la altura dominante (altura promedio de los 10 árboles más altos cada $100 \mathrm{~m}$ de cortina) y la edad, se estimó el índice de sitio (IS) a partir del modelo ajustado para álamo criollo por Peri y Martínez Pastur (1998). Se considera como edad de referencia 40 años, por lo tanto, el IS es la altura dominante de la cortina a los 40 años.

\section{Cuarta Etapa. Instalación de plantaciones demostrativas}

Para la elección de los predios donde instalar las plantaciones demostrativas se procuró cubrir las distintas clases de aptitud y se tuvo en cuenta el interés de los productores, la ausencia de ganado y la accesibilidad.

Quedaron seleccionados cinco predios, uno en sitio apto y cuatro en sitios medianamente aptos. Cuatro de estas plantaciones se realizaron en primavera y, por inquietud de los productores, la restante se realizó en otoño a fin de ver ventajas y desventajas de cada época de plantación.

En base a la altura final de las cortinas en cada clase de aptitud, el cultivo a proteger y la velocidad media del viento durante el período de crecimiento de los cultivos $(8 \mathrm{~m} / \mathrm{s}) \mathrm{se}$ definieron los diseños de las cortinas (Cuadros $\mathrm{N}^{\circ} 2$ y $\mathrm{N}^{\circ} 3$ ).

Se consideró el uso de cortinas principales densas (porosidad óptica menor a 15\%) para la protección de cultivos sensibles al viento (frutilla, lechuga) y cortinas principales 
semipermeables (porosidad óptica entre 15 y 45\%) para cultivos semi-resistentes al efecto del viento (alfalfa, ajo). Las especies utilizadas fueron Populus nigra 'Itálica' (álamo criollo) y Salix matsudana $x$ alba RA 524-43 (Sauce 524-43).

\section{Cuadro $\mathrm{N}^{\circ} 2$}

\section{DISEÑO DE LAS PLANTACIONES INSTALADAS INDICANDO CLASE DE APTITUD, LARGO Y TIPO DE CORTINA, DISTANCIAMIENTOS Y ESPECIES UTILIZADAS.}

\begin{tabular}{|c|c|c|c|c|c|c|}
\hline \multirow{2}{*}{ Productor } & \multirow{2}{*}{$\begin{array}{l}\text { Clase de } \\
\text { aptitud }\end{array}$} & \multirow{2}{*}{$\begin{array}{l}\text { Largo } \\
\text { Cortina } \\
\text { (m) }\end{array}$} & \multirow{2}{*}{$\begin{array}{l}\text { Tipo de cortina / } \\
\text { Número de Cortinas }\end{array}$} & \multicolumn{2}{|c|}{$\begin{array}{l}\text { Distanciamiento } \\
(\mathrm{m})\end{array}$} & \multirow{2}{*}{ Especie } \\
\hline & & & & Filas & Plantas & \\
\hline 1 & $\begin{array}{c}\text { Medianamente } \\
\text { apto }\end{array}$ & 100 & Principal densa doble / 2 & 2 & $\begin{array}{l}2 \\
1 \\
\end{array}$ & $\begin{array}{l}\text { sauce } 524-43 \\
\text { álamo criollo }\end{array}$ \\
\hline 2 & Apto & 150 & $\begin{array}{l}\text { Principal simple } \\
\text { semipermeable / } 1\end{array}$ & & 1,2 & álamo criollo \\
\hline \multirow{2}{*}{3} & \multirow{2}{*}{$\begin{array}{l}\text { Medianamente } \\
\text { apto }\end{array}$} & 100 & $\begin{array}{l}\text { Principal semipermeable } \\
12\end{array}$ & & 1,2 & álamo criollo \\
\hline & & 100 & Secundaria & & 1,5 & sauce $524-43$ \\
\hline \multirow{2}{*}{4} & \multirow{2}{*}{$\begin{array}{l}\text { Medianamente } \\
\text { apto }\end{array}$} & 100 & Principal densa doble / 1 & 2 & $\begin{array}{l}2 \\
1 \\
\end{array}$ & $\begin{array}{l}\text { sauce } 524-43 \\
\text { álamo criollo }\end{array}$ \\
\hline & & 100 & Principal densa doble / 1 & 1,5 & 1 & álamo criollo \\
\hline 5 & $\begin{array}{l}\text { Medianamente } \\
\text { apto } \\
\end{array}$ & 200 & $\begin{array}{l}\text { Principal simple } \\
\text { semipermeable / } 1\end{array}$ & & 1,2 & álamo criollo \\
\hline
\end{tabular}

Previo a la plantación se preparó el sitio con una pasada de subsolador, dos pasadas de arado, dos pasadas de rastra y se realizó el canal de riego con zanjeador. Los hoyos se realizaron con una hoyadora conectada a la toma de fuerza de un tractor. Las plantas utilizadas fueron barbados de 2 años producidos en la zona.

Se aplicó fertilizante (NPK triple 15 en una dosis de $150 \mathrm{~g} /$ planta) en el hoyo de plantación y se colocaron protecciones contra liebres. Estas protecciones consistieron en una red, en forma de tubo, de aproximadamente $50 \mathrm{~m}$ de largo y un diámetro de $18 \mathrm{~cm}$, que se puede cortar al largo deseado. En este caso fueron cortadas a aproximadamente un metro para poder enterrarlas unos $20 \mathrm{~cm}$ y para que tengan una altura sobre el suelo suficiente en caso de nevadas.

Una vez pasado el primer período de crecimiento se realizó una evaluación de las plantaciones instaladas en base al porcentaje de supervivencia y la evaluación de daños por liebre. 


\section{Cuadro $\mathrm{N}^{\circ} 3$ \\ DISTANCIAMIENTO ÓPTIMO ENTRE CORTINAS DENSAS Y SEMIPERMEABLES \\ BASADO EN LA ALTURA DE CORTINAS CORTAVIENTO PARA LAS DIFERENTES CLASES DE APTITUD}

\begin{tabular}{|l|c|c|c|}
\hline Clase de Aptitud & $\begin{array}{c}\text { Altura Final Cortina } \\
(\mathbf{m})\end{array}$ & Tipo de Cortina & $\begin{array}{c}\text { Distanciamiento } \\
\text { Optimo entre Cortinas } \\
(\mathbf{m})\end{array}$ \\
\hline Apto & 25,6 & Densa & 75 \\
\hline Medianamente Apto & 21,5 & Semipermeable & 230 \\
\hline No Apto & 13,7 & Densa & 63 \\
\hline
\end{tabular}

\section{Quinta Etapa. Estudio de viabilidad económica para el establecimiento de plantaciones bajo riego}

Para realizar una apreciación económica de los efectos de la incorporación de cortinas cortavientos, asociadas a otras actividades productivas, se seleccionaron tres alternativas de cultivos existentes en la zona. Estos fueron frutilla y dos rotaciones diferentes; una de ajo, alfalfa y avena y otra de alfalfa y avena. El objetivo de las pasturas, en ambos casos, fue la producción de fardos.

El análisis de los efectos económicos de la incorporación del álamo, se realizó mediante la evaluación de indicadores de rentabilidad aplicados a un flujo de caja incremental. Éste considera solamente los egresos e ingresos monetarios adicionales, en aquellos proyectos productivos agropecuarios que ya se encuentran en marcha sin cortinas de protección (Sapag Chain y Sapag Chain, 1997). Lo que en definitiva se intenta comprobar es la mejora en el rendimiento económico que puede provocar la incorporación de la actividad forestal dentro del esquema productivo actual.

Se consideraron como costos incrementales los costos de instalación de las cortinas, las actividades silviculturales asociadas al mantenimiento de las mismas y los mayores costos de cosecha por incrementos en la producción de los cultivos. Asimismo, se consideraron incrementales los ingresos producidos por la venta de los productos maderables y leña durante el horizonte de planificación, las mejoras en el rendimiento de los cultivos debido al efecto protector de las cortinas y los ingresos extras provenientes de los planes de promoción nacional. El incremento en el rendimiento de los diferentes cultivos, por los efectos de las cortinas protectoras, fue extraído de estudios realizados por Peri y Utrilla (1997), Peri et al. (1998a) y Peri et al. (1998b).

Los indicadores de rentabilidad que se utilizaron para evaluar las diferentes alternativas 
son el Valor Actual Neto (VAN) y la Tasa Interna de Retorno (TIR). Si bien se conocen las deficiencias de la TIR como método de evaluación de proyectos de inversión, se la incorporó para obtener información adicional, y poder conocer la tasa máxima de descuento que aceptaría el proyecto para que este siga siendo rentable (Brealey y Myers, 1993). El criterio de decisión indica que cuando el VAN del Flujo de Caja Incremental arroja resultados positivos se concluirá que es rentable la incorporación de las cortinas dentro del esquema productivo existente.

El horizonte de planificación del proyecto será el que establece la cosecha de la cortina protectora. En este caso se consideró el momento en que el diámetro de los árboles alcanza los $40 \mathrm{~cm}$. Para el sector en estudio se utilizó una tasa de descuento estimada en el 8\%, que representa la rentabilidad mínima que se le exige generalmente a proyectos forestales (Davel, 2008 y Loguercio et al., 2006).

Los flujos de caja para las rotaciones de pasturas y pasturas con ajo se calcularon para una superficie mínima de 5 ha, prorrateando los ingresos y egresos por hectárea. El flujo de caja incremental para la producción de frutillas no se analizó, puesto que para poder llevar adelante la actividad es absolutamente necesario el establecimiento de cortinas protectoras. Es por ello que el estudio se limitó al análisis de los costos de producción y establecimiento del proyecto productivo.

\section{Sexta Etapa. Propuesta de desarrollo forestal para el área y acciones de transferencia.}

Para la propuesta se realizó una simulación de un plan de desarrollo forestal acorde con la actividad socio-económica actual de la zona de estudio y que, por otro lado, utilice como insumo la información producida a lo largo del proyecto. Se reconoce que esta no es la única posibilidad de desarrollo forestal para la región, puesto que el escenario que se pretende recrear representa solamente uno hipotético de todos los posibles. Se consideró una propuesta realista, teniendo en cuenta que actualmente la tasa de forestación en la zona es muy baja.

Una vez definido el escenario, se determinó la cantidad de factores necesarios para llevar adelante el proyecto de desarrollo, la demanda de insumos y mano de obra que esto provocaría, la producción de madera y leña asociada a la actividad forestal, y finalmente, se realizó una estimación de los ingresos netos del conjunto de hectáreas incluidas en el proyecto de desarrollo.

Finalmente, estimada la producción de madera a lo largo del tiempo, se intentó proponer algún aprovechamiento tecnológicamente factible a nivel local para la incorporación de valor agregado al nuevo recurso. Los supuestos asumidos para la simulación son los que siguen:

- $\quad$ No se tuvieron en cuenta las cortinas ya existentes en el área de estudio, las actividades agrícolas que se realizaban en cada predio, ni la estructura de tenencia de tierras.

- $\quad$ Se consideraron las dos clases de aptitud definidas en el área (apto y medianamente apto).

- $\quad$ La distribución de la superficie por actividad y por tipo de cultivo se realizó en base a 
la distribución porcentual actual del uso de la tierra.

- $\quad$ Las dimensiones de los cuadros son las definidas en el estudio de rentabilidad.

- $\quad$ La incorporación a la actividad económica de las tierras se realiza en un lapso de 17 años a una tasa constante a lo largo del tiempo.

- Se eligió como horizonte de planificación de largo plazo 65 años, siendo este el período mínimo en el que se alcanza a realizar una rotación completa de todas las cortinas para las distintas clases de aptitud.

- La propuesta desarrollada es general para el conjunto del área, sin tener en cuenta cada predio individualmente y las actividades programadas anualmente no tienen una localización geográfica determinada.

\section{Transferencia a Productores, Técnicos y Operarios}

Antes de instalar las plantaciones demostrativas se realizaron capacitaciones en plantación a operarios y a los productores dueños de los predios a forestar. En total participaron 18 personas. Además se invitó a todos los productores interesados, a visitar los predios en el momento en que se estaban haciendo las plantaciones demostrativas.

Se realizó un taller al inicio del proyecto para explicar los objetivos y alcances del mismo $y$, al finalizar cada una de las etapas, para informar sobre los resultados parciales obtenidos y las actividades a realizar en la etapa siguiente. En estos talleres participaron productores, técnicos, docentes y estudiantes de la Escuela Agraria, personal del Municipio local y público en general. También se realizó un taller al final del proyecto para discutir la propuesta forestal para el área. Se difundieron los resultados y actividades realizadas durante el proyecto a través de medios televisivos, radiales y gráficos locales. Se elaboró un manual técnico sobre establecimiento y manejo de cortinas forestales para ser entregado a productores y técnicos.

\section{RESULTADOS y DISCUSION}

\section{Primera Etapa. Diagnóstico de la situación socio-productiva de productores con disponibilidad de tierras para el establecimiento de plantaciones bajo riego}

El tamaño promedio de los predios fue de 60,4 ha, siendo el más pequeño de 1 ha y el más grande de 200 ha.

La mayoría de los productores tienen en promedio 50 años de edad, dos de cada tres de ellos viven en el pueblo y poseen título de propiedad de las chacras.

Desde el punto de vista social, se observa que la mayoría de los propietarios desarrollan una vida esencialmente urbana. La ocupación principal se realiza en el pueblo y la actividad rural se encuentra supeditada a los ratos libres o tiempos ociosos. Se prioriza el ingreso seguro producto del trabajo en el sector público (en sus diferentes niveles) y en el sector privado 
(comercio, minería, etc.), y el ingreso predial resulta un plus en algunos casos e incluso a veces es insuficiente para cubrir los gastos en otros. A pesar de esto, el 68\% manifestó arraigo a la actividad, el resto solo mantiene la tierra heredada (Figura $N^{\circ} 1$ ).

\section{Ocupación principal}

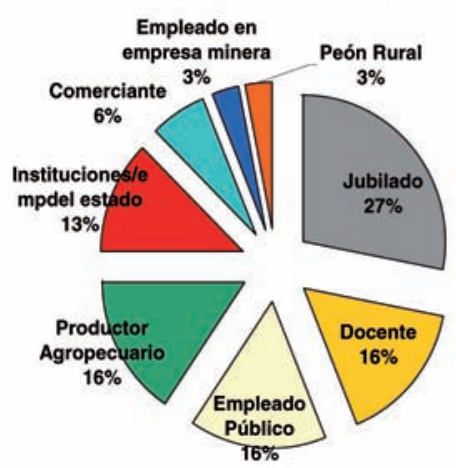

Ocupación secundaria

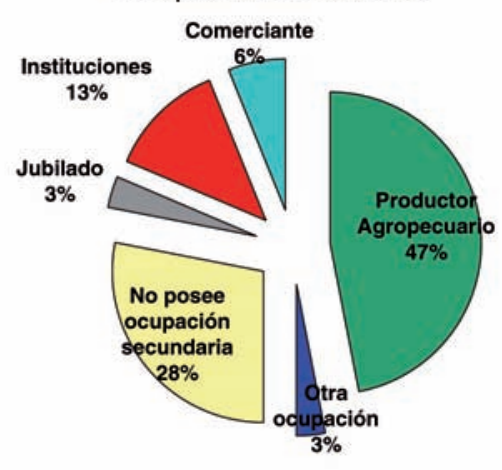

Figura $\mathrm{N}^{\circ} 1$

\section{OCUPACIÓN PRINCIPAL Y SECUNDARIA DE LOS ENTREVISTADOS}

Salvo los casos en los que los entrevistados indican ser productores agropecuarios y para los que el ingreso por esta actividad es la principal fuente de sustento de sus familias, para el resto, el ingreso por la actividad agrícola ganadera representa menos del $40 \%$ del ingreso total.

En Gobernador Gregores la actividad predial tuvo históricamente una fuerte dependencia de la asistencia del estado, que fue quien motorizó mediante programas y estímulos económicos el desarrollo de la actividad agropecuaria.

El perfil productivo de los propietarios entrevistados es ganadero ovino extensivo y en baja escala se cría ganado vacuno, que en algunos casos se combina con la producción de pasturas y hortícola. El 52,2\% de la superficie se destina al pastoreo de animales, el 27,8\% no es aprovechada o permanece improductiva, que sumada a las zonas no aptas, de monte natural y las edificadas, alcanzan aproximadamente al 34,4\% del área de estudio. En el territorio restante $(13,4 \%)$, el suelo se destina a la producción de pasturas y a la actividad agrícola intensiva, como frutales, fruta fina, producción hortícola a cielo abierto y en invernáculos, monte implantado y vivero forestal para la producción de barbados de álamos y sauces.

La producción de pasturas se encuentra relacionada básicamente con la provisión de alimentos para el mismo ganado. Son pocos los productores que tienen producción exclusiva de pasturas con destino a la venta de fardos. En baja escala se cultivan frutas y hortalizas (lechuga, acelga, zanahoria, remolacha, echalotte, papa, tomates, ajo, arvejas y habas), siendo las primeras en su gran mayoría utilizadas como insumo para la producción de dulces y mermeladas. 
Las perspectivas para el sector son positivas, principalmente debido al crecimiento de la demanda local que, desde el 2001, fue creciendo debido al aumento poblacional estimulado por la actividad minera y otras asociadas como obras viales, construcción y servicios. Estas actividades estimularon un proceso migratorio muy importante de otras provincias y países vecinos y se transformaron en el motor de la economía de la zona en los últimos años. Si bien la incorporación de esta nueva población no es bien clara en cuanto a la participación ciudadana y su adaptación al ritmo de vida cotidiano, ocasiona una demanda de alimentos temporalmente creciente, resultando en el principal estímulo de la producción agropecuaria y el fundamento de las buenas perspectivas reinantes para el sector. Los productores planifican incorporar pasturas, aumentar el número de cabezas de ganado y la producción hortícola.

A pesar de contar con una escuela de formación agropecuaria, se observa falta de oferta de mano de obra local para la actividad agropecuaria porque los niveles salariales de las actividades extractivas superan los ofrecidos por los productores. Por un lado, el crecimiento poblacional estimula la demanda de productos agropecuarios, pero por el otro, aumenta la presión inmobiliaria, debido al incremento de valor de las chacras de los alrededores de la localidad. En estos casos, se trata de tierras que ya no estarían disponibles para la producción agropecuaria, estimulando así un proceso de cambio de uso del suelo, con planes de loteo y venta de tierras y/o construcción de viviendas destinadas al alquiler.

El principal problema de infraestructura que obstaculiza el desarrollo del sector es el aislamiento, consecuencia de la red vial existente que dificulta la comercialización de productos y el acceso a la localidad de insumos agropecuarios en forma periódica y a costos razonables. El 65\% de los entrevistados indican que tienen dificultades para acceder a maquinaria. Aquellos que no cuentan con maquinaria suficiente o que no tienen la maquinaria necesaria para realizar alguna actividad, la alquilan por hora al CAP o a la escuela Agropecuaria o bien la comparten con sus vecinos y parientes generalmente de manera informal. La disponibilidad de maquinarias representa una de las mayores fuentes de conflicto entre las Instituciones y los productores.

Otro factor de conflicto es el sistema de riego. Si bien toda el área de estudio posee potencialidad para riego, apenas el $40 \%$ de las zonas aptas para forestar se encuentran sistematizadas. El control y mantenimiento del sistema de riego lo realiza el CAP y si bien no existen restricciones para el uso del agua, los productores señalan pérdidas en la producción por deficiencias del sistema de riego.

Prácticamente no existen agrupaciones privadas de peso que convoquen a los productores. Es por este motivo que pueden comprenderse los fracasos sistemáticos en la organización de actividades de tipo cooperativista y la escasa asociación entre productores que existe en la zona. Los principales obstáculos para su conformación son la pluralidad de objetivos que persiguen, las grandes diferencias socio-económicas que les permite acceder a distintos tipos de conocimientos y la falta de tiempo que pueden destinar a las actividades de intercambio y/o trabajo conjunto. Estas diferencias sociales y los fracasos de tipo cooperativista estimulan el distanciamiento y profundizan las diferencias que hoy ya existen entre los propietarios.

La incorporación del álamo con fines de protección y asociado a cultivos, solo se encuentra algo desarrollada en los productores más dinámicos. Además ya existen en la zona 
viveros que producen barbados, aunque no hay un mercado fuerte y estable conformado en torno a la venta de este producto. El 37,5\% de los propietarios cuentan en sus predios con alguna superficie destinada a la producción de barbados de álamos y sauces, siendo la producción actual de 102.500 plantas por año. El destino de estas plantas es mayormente comercial, vendiéndose en la zona costera (Comandante Luís Piedra Buena, Puerto San Julián y Río Gallegos) y en El Calafate y, la producción restante, la utiliza el mismo productor para la forestación de sus predios. Localmente los productores producen sus propias plantas o utilizan estacas cortadas de árboles de la zona. El principal problema mencionado por los productores son los daños de liebre en las primeras etapas de la plantación.

Existen tanto a nivel nacional como provincial mecanismos de incentivo a las forestaciones para la zona, pero se observó, en general, un desconocimiento de los mismos por parte de los productores.

\section{Segunda Etapa. Determinación del área potencial para forestar con salicáceas y zonificación por clases de aptitud}

Una vez clasificado cada punto del muestreo de suelos, en las distintas clases de aptitud, se redujo la superficie potencial a 2.387 ha (196,6 ha menos que la definida a partir de las imágenes). El área potencial final quedó clasificada de la siguiente manera (Figura №2).

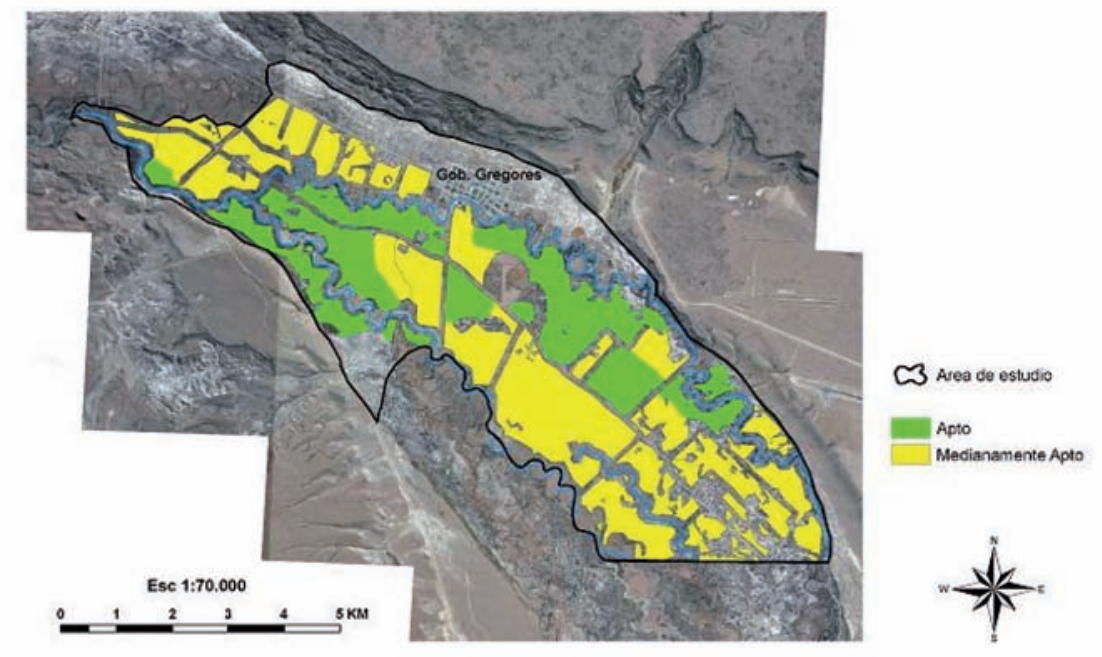

Figura $\mathrm{N}^{\circ} 2$

ZONIFICACIÓN DEL ÁREA POTENCIAL EN SITIOS APTOS (851 ha), MEDIANAMENTE APTOS (1536 ha) Y NO APTOS (1961 ha) PARA EL CULTIVO DE SALICÁCEAS 
Tercera Etapa. Crecimiento de las plantaciones existentes dentro del área potencial y para cada clase de aptitud de sitio

Las diferencias más importantes, entre las distintas clases de aptitud, se presentan en los valores de IS y volumen, donde se observa una tendencia que responde a la zonificación realizada. Esta tendencia también se observa en el crecimiento en altura, disminuyendo de los sitios aptos a los no aptos. En cuanto a los valores de crecimiento en diámetro, estos solo se presentan como información adicional ya que influenciados, principalmente, por el distanciamiento de los árboles en la cortina. Si bien se midió una sola cortina, hay que resaltar los bajos valores obtenidos en el sitio clasificado como no apto (Cuadro $\mathrm{N}^{\circ} 4$ ).

\section{Cuadro $\mathrm{N}^{\circ} 4$ \\ VALORES PROMEDIOS DE EDAD, ÍNDICE DE SITIO (IS) Y CRECIMIENTO POR AÑO EN DAP, ALTURA Y VOLUMEN EN LAS CORTINAS DE ÁLAMO CRIOLLO MEDIDAS EN LAS TRES CLASES DE APTITUD}

\begin{tabular}{|c|c|c|c|c|c|}
\hline Clase de Aptitud & $\begin{array}{c}\text { Edad } \\
\text { (años) }\end{array}$ & IS $_{40}$ & $\begin{array}{c}\text { Crec. Medio DAP } \\
\text { (cm/año) }\end{array}$ & $\begin{array}{c}\text { Crec. Medio Altura } \\
\text { (m/año) }\end{array}$ & $\begin{array}{c}\text { Crec. Medio Volumen } \\
\text { (m3/100m de cort./año) }\end{array}$ \\
\hline Apto & 50 & 23 & 1,0 & 0,50 & 4,94 \\
Medianamente apto & 37 & 20 & 0,9 & 0,43 & 2,15 \\
No Apto & 20 & 14 & 0,4 & 0,30 & 0,10 \\
\hline
\end{tabular}

En cuanto a las especies presentes en el área y en base a los estudios existentes en la zona (Peri et al., 1997 y Peri y Monelos, 1998) y las mediciones realizadas en el presente proyecto, los mejores comportamientos, en los sitios medianamente aptos, los presentaron Salix matsudana $x$ alba RA 524-43 y Populus nigra 'Itálica'. Mientras que para los sitios aptos, a los dos anteriores se le suman Populus nigra 'Moissac', Populus x canadensis 'I-214' (solo en sitios protegidos) y un cultivar de Populus trichocarpa que no se pudo identificar. Los valores de crecimiento para estas últimas especies van de 0,5 a $1 \mathrm{~cm} /$ año en diámetro y alrededor de los $0,5 \mathrm{~m} / \mathrm{año} \mathrm{en} \mathrm{altura.}$

\section{Cuarta Etapa. Plantaciones demostrativas.}

Las plantaciones realizadas en primavera fueron evaluadas en mayo del presente año, luego de pasado el primer período de crecimiento. Los resultados se presentan en el Cuadro $N^{\circ} 5$. La plantación realizada en otoño se evaluará recién en otoño de 2011. 


\section{Cuadro $N^{\circ} 5$ \\ RESULTADOS DE SOBREVIVENCIA PARA LAS CORTINAS DEMOSTRATIVAS INSTALADAS}

\begin{tabular}{|c|c|c|c|}
\hline Productor & Cortina & Especie & $\begin{array}{c}\text { Supervivencia } \\
(\%)\end{array}$ \\
\hline \multirow{2}{*}{1} & 1 & $\begin{array}{c}\text { álamo criollo } \\
\text { sauce 524-43 }\end{array}$ & $\begin{array}{c}66 \\
96\end{array}$ \\
\cline { 2 - 4 } & 2 & $\begin{array}{c}\text { álamo criollo } \\
\text { sauce 524-43 }\end{array}$ & $\begin{array}{c}49 \\
54\end{array}$ \\
\hline \multirow{2}{*}{2} & 1 & álamo criollo & 94 \\
\hline \multirow{3}{*}{3} & 1 & álamo criollo & 32 \\
\cline { 2 - 4 } & 2 & álamo criollo & $\mathbf{8 4}$ \\
\cline { 2 - 4 } & 3 & sauce 524-43 & 44 \\
\hline \multirow{2}{*}{4} & 1 & álamo criollo & 90 \\
& 2 & sauce 524-43 & $\mathbf{9 0}$ \\
\cline { 2 - 4 } & \multirow{2}{*}{2} & álamo criollo & $\mathbf{8 3}$ \\
\hline
\end{tabular}

Las cortinas de los productores 2 (sitio apto) y 4 (sitio medianamente apto) tuvieron riego adecuado desde sus inicios y los valores de prendimiento fueron muy aceptables. Los productores 1 y 3 (sitios medianamente aptos) no realizaron los riegos adecuadamente y los prendimientos son menores, observándose en ambos casos valores altos de supervivencia en una de las cortinas y bajos en las restantes.

En cuanto a las protecciones contra liebres, estas resultaron efectivas hasta el momento de la evaluación, no observándose daños. En la plantación del productor 3 , algunas de las protecciones colocadas en los sauces se "volaron" por los fuertes vientos y, de las plantas que quedaron sin protección, ya el $21 \%$ presentaban daños por liebres.

\section{Quinta Etapa. Estudio de viabilidad económica para el establecimiento de plantaciones bajo riego}

Analizados los flujos de caja incrementales para las rotaciones propuestas, se observa que el VAN resulta positivo en todos los casos, con lo cual la incorporación de cortinas cortavientos permite aumentar la rentabilidad predial actual. Los mejores resultados se obtienen en los casos en los que también se incorporan cortinas secundarias. Estas permiten mejorar la producción de los cultivos asociados en menos tiempo y así obtener márgenes más rentables. La tasa interna de retorno, en los predios con cortinas, siempre supera el $25 \%$ con respecto de la situación sin cortinas protectoras (Cuadro $\mathrm{N}^{\circ} 6$ ). 


\section{Cuadro $N^{\circ} 6$ \\ INDICADORES DE RENTABILIDAD PARA LAS ROTACIONES DE ALFALFA - AVENA Y AJO - ALFALFA - AVENA}

\begin{tabular}{|l|c|c|c|c|}
\hline \multirow{2}{*}{ VAN Incremental } & \multicolumn{2}{|c|}{ Alfalfa - Avena } & \multicolumn{2}{c|}{ Ajo - Alfalfa - Avena } \\
\cline { 2 - 5 } & $\begin{array}{c}\text { Con Cortina } \\
\text { Secundaria }\end{array}$ & $\begin{array}{c}\text { Sin Cortina } \\
\text { Secundaria }\end{array}$ & $\begin{array}{c}\text { Con Cortina } \\
\text { Secundaria }\end{array}$ & $\begin{array}{c}\text { Sin Cortina } \\
\text { Secundaria }\end{array}$ \\
\hline Sitios Aptos & $14.509,3$ & $9.159,3$ & $11.890,8$ & $7.574,4$ \\
\hline Sitios Moderadamente a Poco Aptos & $13.864,4$ & $8.595,4$ & $11.772,3$ & $7.533,5$ \\
\hline TIR del Proyecto Incremental & & & & \\
\hline Sitios Aptos & $38,5 \%$ & $35,7 \%$ & $39,8 \%$ & $36,9 \%$ \\
\hline Sitios Moderadamente a Poco Aptos & $27,9 \%$ & $26,9 \%$ & $27,8 \%$ & $26,8 \%$ \\
\hline
\end{tabular}

Si se compara las dos rotaciones analizadas, los indicadores muestran que es más rentable la rotación de pasturas exclusivamente. La incorporación de cortinas cortavientos es aún más rentable en el caso de sitios aptos.

Para el cultivo de frutillas se estimaron solo los costos e ingresos relacionados a la instalación de las cortinas densas. En este caso la instalación de las cortinas es una condición limitante para la producción, no se puede producir frutillas sin la presencia de cortinas de protección.

\section{Sexta Etapa. Propuesta de desarrollo forestal para el área.}

Siguiendo los supuestos del modelo de simulación propuesto, se calcularon los costos, ingresos, insumos y la producción forestal resultado de las actividades silviculturales que deben realizarse anualmente durante el horizonte de planificación.

Durante los primeros 17 años se incorporan a la producción 100 ha/año, correspondiendo 92 ha a rotaciones de pasturas y pasturas con ajo y 8 ha a cultivos fruto hortícolas. Esto implica un ritmo de forestación constante de 11.000 m de cortinas por año, que demandarán aproximadamente 10.000 plantas por año, cantidad que puede cubrirse con la producción local. Posteriormente la tasa de forestación disminuye ya que solo se reemplazarán las cortinas secundarias que se irán cortando a partir del año 18. Luego de cubrirse una superficie de 1.700 ha se manejará el rebrote de las cortinas principales por un turno, por lo que no será necesario volver a plantar en los últimos años de la planificación.

En función de los planes de manejo establecidos, se deben realizar podas de formación. Para el caso de cortinas semipermeables, instaladas en sitios aptos, se realizan a los 2 y 4 años y en sitios medianamente aptos a los 3 y a los 5 años. Las cortinas densas requieren la realización de las podas a los 2 y 4 años en sitios aptos y a los 2, 4 y 6 años en sitios medianamente aptos. Las podas de fuste hasta $2-2,5 \mathrm{~m}$, en sitios aptos, se realizan en las cortinas densas a los 11 años y en las semipermeables a los 6 años, mientras que, en sitios medianamente aptos, se prevé su realización a los 7 años para ambos tipos de cortina. 
Los turnos de corta para las cortinas densas son de 46 años en los sitios aptos y de 50 años en los medianamente aptos y, para las cortinas semipermeables, de 36 años en sitios aptos y de 40 años en los medianamente aptos.

El modelo propuesto tiene un requerimiento promedio de 1.500 horas/máquina/año para las tareas de preparación de sitio, plantación y mantenimiento de canales de riego. Dada la estacionalidad de las actividades a realizar, que en el caso de la plantación se restringe a los meses de abril-mayo y agosto-septiembre y, que el mantenimiento de canales debe realizarse antes del período de riego, la maquinaria puede transformarse en un cuello de botella para un proyecto de desarrollo forestal de estas dimensiones. En esta situación, habría que buscar algún tipo de solución, ya sea incorporando más maquinaria o realizando algún pool entre productores para la compra de las mismas.

Sin tener en cuenta la mano de obra de la maquinaria que se alquila por hora y las tareas de aprovechamiento que se supuso tercerizadas, la actividad forestal por sí sola no genera, en este caso, una gran cantidad de puestos de trabajo. La mayor demanda de mano de obra estará ligada a las actividades asociadas, como por ejemplo tractoristas, el transporte, el aprovechamiento y las actividades agrícolas y ganaderas. Por otro lado, si la madera producida se procesara en la localidad, podría instalarse un aserradero fijo que daría empleo permanente a entre 10 y 15 personas.

La producción de leña proveniente de la corta de las cortinas secundarias ronda los 500 m3 estéreo desde el período 17 hasta el 34. A partir del período 35 se incorpora la leña de álamo de las cortinas principales que se comienzan a aprovechar y se mantiene constante a futuro en alrededor de 2.500 m3/año. La producción estimada de madera rolliza aserrable comienza obtenerse a partir del período 35, estabilizándose a partir de los 39 años en un promedio de $6.500 \mathrm{~m} 3 /$ año.

Desde el período 2 y hasta el período 18, se producen ingresos provenientes de los subsidios para el establecimiento de forestaciones bajo riego promocionados por la Nación (Ley 25.080). En este escenario se alcanzaría un ingreso anual de alrededor de \$47.300. A partir del año 17 comienzan a percibirse los ingresos por la venta de leña, que hasta el año 34 proporciona ingresos anuales de $\$ 25.000$. Cuando comienza a obtenerse madera rolliza, el ingreso anual por la venta de esta más la leña, alcanza en promedio $\$ 900.000$.

Durante los primeros años los costos varían alrededor de los $\$ 160.000$ por año, para estabilizarse a partir del año 43 en $\$ 100.000$ anuales. Las tareas que demandan mayor nivel de gasto son las que requieren el uso de maquinaria. En este caso, el mantenimiento de los canales de riego y la plantación.

Si se compara los ingresos con los gastos, puede observarse que la actividad forestal, sin tener en cuenta los ingresos de las actividades asociadas, requiere inversiones durante los primeros 34 períodos, hasta que la venta de madera comienza a aportar ingresos que superan ampliamente los costos durante el resto del horizonte de planificación. La mejora en los ingresos de las actividades asociadas ayudará a recuperar parte de la inversión necesaria para la instalación de las cortinas durante los primeros años. 


\section{CONCLUSIONES}

El desarrollo forestal del valle de Gobernador Gregores es posible y redundaría en beneficios para la actividad agropecuaria local, debido a que:

- La instalación de cortinas forestales, por las condiciones climáticas de la zona, es imprescindible y de fundamental importancia para mejorar la productividad del valle. Para ello existe una superficie de 2.387 ha aptas para forestar con salicáceas.

- Las cortinas instaladas no solo aumentarían la producción agropecuaria sino también brindarían otros beneficios adicionales, como madera y leña, protección del suelo, embellecimiento del paisaje, mejora de la calidad de vida de los pobladores, aumento del valor inmobiliario y generación de empleo.

- Existen mecanismos de promoción para la actividad forestal a nivel nacional, que consisten en montos no reintegrables que se entregan a los productores que forestan y que alcanzan a cubrir gran parte de los costos de forestación.

- Existen estudios para la zona que demuestran un importante incremento de la producción agrícola con la protección de las cortinas. Además, en el presente proyecto, se cuantificó como se incrementa la rentabilidad de un predio al incorporar las cortinas de protección con respecto al mismo predio sin cortinas.

- Un aspecto positivo para el sector es la demanda local creciente de alimentos debido al incremento poblacional. Además la actividad podría generar una salida laboral para los estudiantes y egresados de la Escuela Agraria y, por otro lado, se generaría mayor estabilidad para los trabajadores rurales, puesto que los cultivos se realizan generalmente durante el verano y la actividad forestal en el otoño-invierno.

- Quedaron definidas durante el proyecto las especies a utilizar en cada sitio, el tipo de cortinas a instalar, los distanciamientos entre plantas y entre cortinas, los cuidados a tener en cuenta durante y después de la plantación y los posibles productos forestales a obtener. Estos conocimientos fueron transmitidos a productores y técnicos a través de charlas, talleres, plantaciones demostrativas, participación en programas radiales y televisivos locales y entrega de informes y un manual sobre establecimiento de cortinas. Además existe experiencia local en la producción de plantas y la cantidad producida alcanza para cubrir la propuesta realizada.

- En los últimos años se triplicó la población de Gobernador Gregores, esto se debió principalmente a la actividad minera. Esta actividad se basa en un recurso no renovable, por lo tanto, limitado en el tiempo. La actividad agrícola asociada a la forestal se presentan como una alternativa válida y sustentable para paliar los efectos de la finalización de la actividad minera.

- Si se llevara a la práctica la propuesta realizada, no solo mejoraría la producción agrícolaganadera de la zona, sino que además se generaría un nuevo recurso renovable, que podría utilizarse con fines industriales y/o energéticos. 
Para lograr el desarrollo propuesto deben mejorarse ciertos aspectos y hay que tener en cuenta algunos inconvenientes:

- Existe infraestructura de riego para gran parte del área, lo que resulta una ventaja, sin embargo se debería mejorar y regularizar su funcionamiento para que el agua llegue a los predios en el momento oportuno. Por otro lado tendría que haber un control sobre el uso del agua y se debería evaluar si un canon de riego ayudaría a mejorar la eficiencia en el uso del sistema.

- La maquinaria utilizada por la mayoría de los productores es alquilada principalmente al CAP. Esta maquinaria es en gran parte antigua y no alcanzaría a cubrir la demanda para el desarrollo propuesto. Si bien no es sencillo, aparece como una alternativa válida pensar en la organización y unión de productores para la compra de maquinaria. Para ello el Estado podría diseñar una línea de crédito a baja tasa.

- Hay dificultades para comercializar los productos fuera del pueblo por las distancias y las condiciones de los caminos, pero actualmente se están asfaltando las rutas que comunican a Gregores con otras poblaciones de la costa y de la cordillera.

- La mano de obra para la actividad agropecuaria es escasa y debe competir en desventaja con otras actividades como la minería.

- Los productores que viven de las chacras son muy pocos, la mayoría tiene a la actividad agropecuaria como algo secundario. Esto dificulta la asociación entre productores y hace que las decisiones productivas sean muy dependientes de las políticas del Estado.

- Debido al crecimiento poblacional hay demanda inmobiliaria sobre las tierras que rodean la ciudad. Esto va a producir un cambio de uso en las mismas.

- Para el desarrollo forestal del área se debe realizar una mayor inversión en los primeros años, que recién es recuperada ampliamente a partir del año 40. Los mayores costos del inicio podrían ser recuperados a través de una mejora de la producción asociada y los planes de promoción estatales. 


\section{REFERENCIAS}

Amico, I., 2001. Viverización y cultivo de álamos y sauces. INTA. 47p.

Brealey, R. y Myers, S., 1993. Fundamentos de financiación empresaria. Editorial Mc GrawHill.

Davel, M., Havrylenko, S. y Barbé, A., 2007. Informe final del proyecto: "Estudio exploratorio para el desarrollo de forestaciones de salicáceas en tres zonas de la Patagonia". SECyT Programas y Proyectos Especiales, CIEFAP, Fundación para el Desarrollo Forestal, Ambiental y del Ecoturismo Patagónico. 140p.

Davel, M., 2008. Establecimiento y manejo del pino oregón en Patagonia (Ed). Capítulo 9. Determinación del turno financiero en plantaciones de pino oregón en diferentes calidades de sitio (Fernández M.V.). Manual № 9. CIEFAP. 111-129.

Deschamps, J., y Wright, J.,1997. Patología forestal del cono sur de América. Orientación Gráfica Editora S.R.L. Buenos Aires. 237 p.

FAO, 1980. Los álamos y los sauces. Colección FAO Montes №10. Roma. 349p.

García, J., 2002. Forestación con salicáceas en áreas bajo riego de Patagonia. SAGPyA. NEF Patagonia. 36 p. Extraído de http://www.sagpya.mecon.gov.ar/new/0-0/ forestacion/deleg/ manusali.pdf

Loguercio, G., Fernández, M. V., Ruiz Tagle-Molina, M., Davel, M. y Havrylenco, S., 2006. Modelo de Asignación de Recursos para la generación de Cuencas de Forestación en la provincia del Chubut. Informe Final Proyecto PIA 12/04. Proyecto Forestal de Desarrollo de la SAGPyA - CIEFAP. 129 p.

Menoyo, H., Mombelli, O., y Davel, M., 1994. Los álamos en la Patagonia. Serie Técnica 2. Ed. Universitaria de la Patagonia. $41 \mathrm{p}$.

Peri, P., Monelos, L. y Heinz, E., 1997. Ensayos de introducción de clones de salicáceas en la provincia de Santa Cruz. II Congreso Forestal Argentino y Latinoamericano. Tomo 4. Del 13 al 15 agosto. Posadas-Misiones.

Peri, P.L. y Utrilla, V., 1997. Efectos de cortinas cortaviento sobre la producción de alfalfa (cv Dawson) en la provincia de Santa Cruz, Argentina. Actas II Congreso Forestal Argentino y Latinoamericano. Tomo Política, Economía y Educación. Posadas, Misiones, 13-15 de Agosto de 1997. Pp. 59-65.

Peri, P., y Martínez Pastur, G., 1998 Crecimientos en cortinas cortaviento de Populus nigra cv Itálica en Patagonia Sur (Argentina) Rev. Investigación Agraria. Sistemas y Recursos Forestales 7 (1-2): 73-83. España.

Peri, P., y Monelos, L., 1998. Ensayo de introducción de clones de salicáceas en Patagonia Sur. Actas Primer Congreso Latinoamericano de IUFRO. Tema 2 (14): "Sistemas Integrados de Producción y Desarrollo Rural". Valdivia, Chile. 22-28 de Noviembre de 1998.

Peri, P., Cittadini, E., Espina, H. y Romano, G., 1998a. Incidencia del efecto protector de cortinas forestales en la producción de frutilla variedad Fern en Santa Cruz, Argentina. Actas 
primer Congreso Latinoamericano de IUFRO Tema 2 (13). Valdivia, Chile. Publicado en CD.

Peri, P., Cittadini, E. y Romano, G., 1998b. Efecto de cortinas cortaviento sobre la producción de ajo violeta en la provincia de Santa Cruz, Argentina. Actas Primer Congreso Nacional de Profesionales de Cambio Rural. Buenos Aires, 20-21 de mayo de 1998. Acta de Resúmenes Pp. 99. Trabajo completo en WEB: www.inta.gov.ar/cambio/links/PC243.PDF

Riu, R., Arreghini, R., y Pérez Valenzuela, R., 1988. Influencia del factor edáfico en el crecimiento de P. x euroamericana cv I-214 en el departamento San Martín - Mendoza. VI Congreso Forestal Argentino. Tomo 2. 16 al 20 de agosto de 1988. santiago del Estero. Ed. El Liberal. P. 429-432.

Sapag Chain, Nassir y Sapag Chain, Reinaldo, 1997. Preparación y Evaluación de Proyectos. 3ra Edición. Editorial Mc Graw Hill.

Suarez, R., 1993. Las salicáceas. Congreso Forestal Argentino y Latinoamericano. Comisión VI. Paraná, Entre Ríos, 1993. AFoA. 